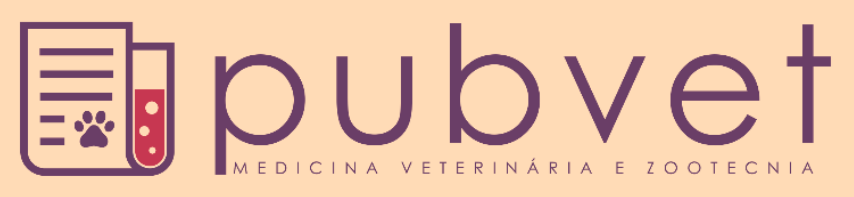

https://doi.org/10.31533/pubvet.v14n1a494.1-5

\title{
Urolitíase e hidrometra em cadela: relato de caso
}

\section{Laide Danielle Coelho da Silva Chaves ${ }^{1 *} \bullet$, Francisco Lima Silva ${ }^{2}$, , Jacqueline Maria Canuto de Sousa $^{30}$, Joice Rayane de Alencar Oliveira ${ }^{30}$, Catarina Rafaela Alves da Silva ${ }^{3}$, Mônica Sousa Holanda ${ }^{4}$, Laíz Pinheiro Santos ${ }^{4}$}

\footnotetext{
${ }^{I}$ Discente em do curso de Medicina Veterinária em Universidade Federal do Piauí. Teresina-PI Brasil.

${ }^{2}$ Professor em Universidade Federal do Piauí, área de Medicina Veterinária. Teresina-PI Brasil.

${ }^{3}$ Médico veterinário. Teresina-PI Brasil.

${ }^{4}$ Discente em do curso de Medicina Veterinária em Universidade Federal do Piauí. Teresina-PI Brasil.

*Autor para correspondência, E-mail: la_danielle@hotmail.com
}

\begin{abstract}
Resumo. A hidrometra e urolítiase em cães são enfermidades muito comuns na medicina veterinária, em que fatores como alimentação, predisposição genética e até mesmo a idade são predisponentes para tais ocorrências, sendo a intervenção cirúrgica a mais indicada como forma terapêutica. Dessa forma, este trabalho teve como objetivo relatar um caso clínico de uma cadela acometida por cálculo vesical e hidrometra, apresentando sinais clínicos inespecíficos e específicos, sendo realizado exames físicos e laboratoriais para diagnóstico e submetida a cirurgia na cidade de Teresina, Piauí.
\end{abstract}

Palavras-chave: Urinária, renal, piometra

\section{Urolithiasis and hydrometra in bitch: case report}

\begin{abstract}
Hydrometra and urolithiasis in dogs are very common diseases in veterinary medicine, in which factors such as food, genetic predisposition and even age are predisposing to such occurrence, and surgical intervention is the most indicated as a therapeutic form. Thus, this study aimed to report a clinical case of a female dog affected by bladder and hydrometra calculus, presenting nonspecific and specific clinical signs, being performed physical and laboratory examinations for diagnosis and undergoing surgery in the city of Teresina, Piauí.
\end{abstract}

Keywords: Urinary pyometra, renal

\section{Urolitiasis y hidrómetra em hembra canina: reporte de um caso}

Resumen. La urolitiasis e hidrómetra en los perros son enfermedades muy comunes en la medicina veterinaria, en las que factores como los alimentos, predisposición genética e incluso la edad predisponen a tales fatos, siendo la intervención quirúrgica la más indicada como forma terapéutica. Por lo tanto, este estudio tuvo como objetivo informar un caso clínico de una perra afectada por cálculos de vejiga e hidrómetra, presentando signos clínicos inespecíficos y específicos, realizando exámenes físicos y de laboratorio para el diagnóstico y sometida a cirugía en la ciudad de Teresina, Piauí.

Palabras clave: Piometra, urinaria, renal

\section{Introdução}

Uma das grandes enfermidades e desafios do trato urinário na clínica de pequenos animais advém das urolitíases (Koehler et al., 2009), que podem chegar até a causar obstruções (Grauer, 2015; $\underline{\text { Syme et }}$ al., 2006). São concentrações definidas como massas de formas esferóides, ovóides ou facetadas, 
formadas por precipitações sucessivas de sais de ácidos inorgânicos, substâncias orgânicas ou também de outros elementos, estas nomeadas de acordo com a sua composição cristalina, assim como com a sua localização (Ettinger et al., 2002; Ettinger et al., 2017; Osborne et al., 1999a). A etiologia da urolitíse depende de vários fatores podendo se formar de várias formas, assim também dependendo do tipo de urólitos envolvido. Podem ser formados como resultado da precipitação de íons calculogênicos em condições de supersaturação urinária; em decorrência de lesão ou inflamação renal; como consequência de estase urinária etc (Bartges, 2012; Ling et al., 1998).

A remoção cirúrgica dos urólitos é um método muitas vezes invasivo e mutilante, embora havendo opções cirúrgicas menos invasivas assim inicialmente recorre-se a tratamentos alternativos como opções voltadas ao bem-estar animal e com menores níveis de morbidade (Raditic, 2015).

A hidrometra é uma alteração uterina que leva ao acúmulo de líquido dentro do órgão. O útero das cadelas sofre alterações durante todo o seu ciclo estral, são mudanças morfológicas sob a influência dos seus hormônios (Oliveira et al., 2008). O período diestro normal da cadela não prenha dura em média 70 dias, nesse período seu útero está em constante influência de progesterona produzida pelos corpos lúteos ovarianos (Fossum, 2014; Nelson \& Couto, 2015). Além disso, outras etiologias estão associadas à obstrução da cérvix ou vagina, o hiperestrogenismo exógeno e a persistência do hímen, causando assim o acúmulo de líquido no útero (Mialot, 1984; Nascimento \& Santos, 2000).

O presente trabalho tem como objetivo relatar um caso clínico/cirúrgico de um paciente que apresentou nefrolitíase e hidrometra.

\section{Relato de caso}

Um canino, fêmea, sem raça definida, sete anos, foi levada para a Clínica Veterinária Animal's na cidade de Teresina, Piauí, pois apresentava apatia, sangue na urina um pouco mais de uma semana, segundo o tutor, falta de apetite, magreza, mucosas hipocoradas e disúria, sendo realizado o análise clínico e submetida aos exames complementares.

No exame físico apresentou temperatura dentro da normalidade, discreta dor abdominal, e o animal urinou com coloração avermelhada. O paciente foi submetido imediatamente aos exames de imagem, como ultrassonografia e raio-X.

No hemograma e bioquímico não houve alteração significativa. No exame ultrassonográfico foi constatado espessamento do útero, presença de cálculos na bexiga e partir do exame feito no raio-x com contraste, foi confirmado a presença de urólitos (Figura 1).

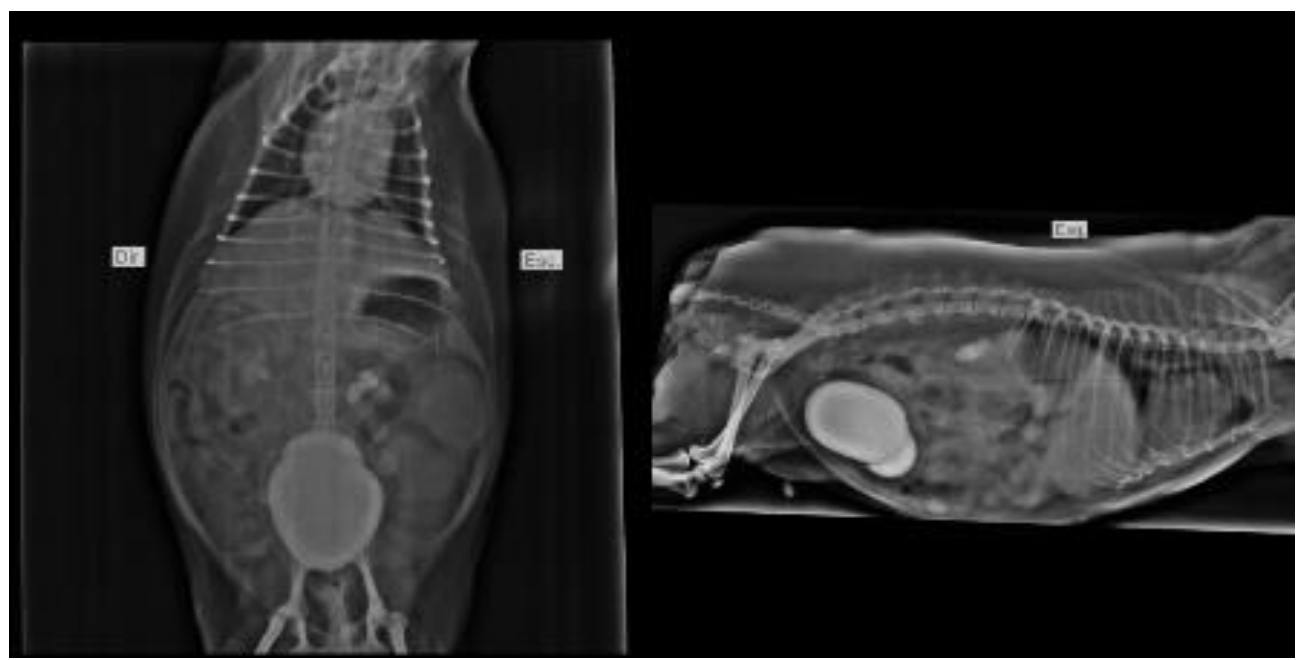

Figura 1. Radiografia com contraste permitindo a visualização de cálculo na bexiga de uma cadela.

O animal foi encaminhado em seguida para a cirurgia para retirada de urólitos e ovariohisterectomia total. Durante a cirurgia, foram retirados dois urólitos e a partir da OSH constatou-se que se tratava de uma hidrometra a partir da retirada do útero (Figura 2). Após o procedimento cirúrgico, o animal não resistiu e veio a óbito. 


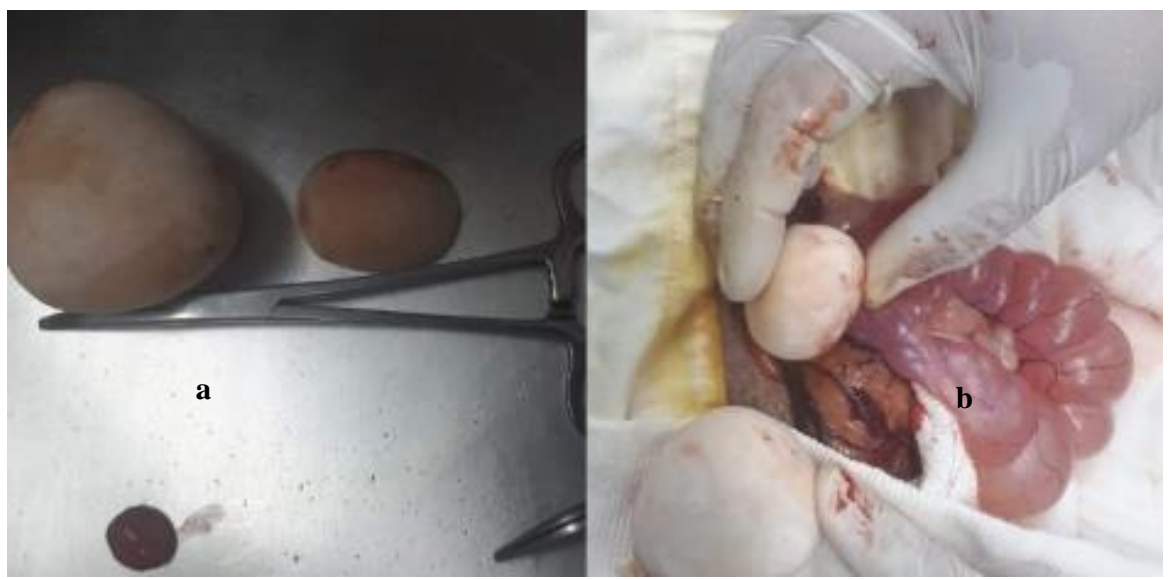

Figura 2. Cálculos retirados da bexiga (a) e útero (b) de uma cadela

\section{Discussão}

A progesterona é um hormônio que estimula a atividade secretora das glândulas endometriais e reduz a atividade do miométrio no útero. Diante disso, pode ocorrer o acúmulo de líquido na luz uterina das glândulas endometriais caracterizando a hidrometra e a mucometra nas fases iniciais, o que pode levar ao acúmulo de bactérias ascendentes a partir da vagina, originando a piometra (Fossum, 2014). O paciente relatado apresentou sinais de hidrometra mediante a ausência de muco e presença somente de líquido a partir da incisão uterina realizada após a cirurgia. A partir dos sinais clínicos e exames complementares confirmou-se o diagnóstico de urolítiase, já a hidrometra houve suspeita a partir do exame de ultrassonografia sendo confirmado após ovariohisterectomia. $\mathrm{O}$ animal relatado não apresentou sintomas característicos de piometra, como secreção vaginal, febre e alterações no hemograma como leucocitose.

Como medicação pré-anestésica foi administrada a associação de tiletamina com zolazepam $(0,1$ $\mathrm{mg} / \mathrm{kg}$, IV) e sulfato de morfina $(0,3 \mathrm{mg} / \mathrm{kg}$, IM). A indução anestésica constou de diazepam $(0,5 \mathrm{mg} / \mathrm{kg}$, IV) e tiopental sódico $\left(2,5 \mathrm{mg} / \mathrm{kg}\right.$, IV) e manutenção à base de isoflurano vaporizado em $\mathrm{O}_{2}$ a $100 \%$ em circuito semiaberto. A cirurgia do presente caso teve como objetivo realizar a laparoscopia para investigação do espessamento uterino identificado no ultrassom, sendo a OSH utilizada como meio terapêutico. Em relação à celiotomia, optou-se pelo acesso convencional de remoção do útero, considerando o potencial risco de contaminação intrauterina o que pode alcançar a sessão intracavitária do órgão.

A técnica utilizada na OSH foi a de Stone (2007) feita a incisão retroumbilical, em seguida feito a identificação do corno uterino direito com o auxílio do afastador de Farabeuff. O útero foi identificado com espessamento de volume, sendo retirado e feito uma incisão posteriormente e sendo verificado líquido dentro de sua cavidade.

Os sinais clínicos demonstrados pela paciente canina como incontinência urinária e hematúria são compatíveis com cistite e afecções de trato urinário inferior (Waki et al., 2010). A formação da urolitíase está relacionada à precipitação de sais dissolvidos em urina supersaturada, gerando cristalização em consequência da supersaturação urinária. Quando os cristais se agregam formam os cálculos (Grauer, 2015; Grauer, 2005).

Os cálculos urinários possuem a classificação conforme a sua composição mineral, formato e localização no trato urinário. Estruvita, oxalato de cálcio, urato, misto, silicato e cistina são os principais cálculos urinários relatados em cães e gatos (Waki et al., 2010). No presente relato, não houve realização no paciente acima descrito de exame chamado urinálise, sendo encaminhado diretamente para a cirurgia para a retirada do cálculo da bexiga, não havendo descrição do tipo de cálculo que se tratava.

As fêmeas caninas são mais propensas à formação destes cálculos em comparação aos machos, principalmente pelo motivo da maior susceptibilidade às infecções do trato urinário devido à anatomia uretral da fêmea, que é mais curta e larga, proporcionando uma entrada facilitada de bactérias de forma ascendente (Houston et al., 2004). 
Cálculos grandes, infecção urinária ou obstruções, associados à ocorrência de oxalato de cálcio, fosfato de cálcio e silicato, deve ser realizada cistotomia, a qual consiste na remoção manual dos cálculos através de método cirúrgico invasivo. No entanto, não é necessária a remoção dos cálculos em pacientes assintomáticos (Houston et al., 2004; Koehler et al., 2009; Latney \& Donnelly, 2013).

O diagnóstico de urolitíase é realizado a partir da anamnese, exame físico e exames complementares. Os sinais clínicos observados no animal são inespecíficos, como foi o caso da cadela mencionada no relato acima, dentre os quais podem ser destacados a hematúria, estrangúria, disúria, polaciúria, incontinência urinária e mudança comportamental (Koehler et al., 2009; Osborne et al., 1995; Osborne et al., 1999b).

Apesar de não ter sido feito a urinálise, no presente caso, vale ressaltar que se trata de um exame realizado para indicar a inflamação do trato urinário demonstrando piúria, hematúria, bacteriúria, aumento da quantidade de células epiteliais e proteinúria, assim como o $\mathrm{pH}$ urinário sugerindo o tipo de cálculo presente. A cultura microbiológica e antibiograma são importantes para a avaliação de qualquer possível infecção urinária concomitante à urolitíase, no entanto, também não foi realizada (Grauer, 2015).

A radiografia simples não detecta urólitos de tamanho menor que dois $\mathrm{mm}$ de diâmetro, pode evidenciar cálculos formados por oxalato de cálcio e estruvita, por se mostrarem radiopacos. A técnica de ultrassonografia é segura e sensível para o diagnóstico (Osborne et al., 1995).

$\mathrm{O}$ tratamento se restringiu à remoção cirúrgica já que não podem ser dissolvidos. Entretanto, dietas não acidificantes contendo baixos teores de cálcio e oxalato, bem como quantidade adequada de cálcio, assim como a quantidade de fósforo adequada para evitar a ativação renal de vitamina D. A cistotomia foi realizada com o intuito de remoção do urólito diagnosticado pelo raio- $\mathrm{x}$, sendo observado a presença de dois urólitos. A prevenção da ocorrência de urólitos se resume em promover a diurese, através da maior ingestão de água, erradicação de possíveis infecções do trato urinário, aumentar a solubilidade, reduzir a concentração de cristaloides e sugerir ao proprietário que introduza uma dieta apropriada para evitar a formação de cálculos (Ettinger et al., 2017). Apesar de a cirurgia ter sido realizada imediatamente após o diagnóstico, o animal não resistiu e teve parada cardiorrespiratória, vindo a óbito após o término da operação.

\section{Conclusão}

O relato de caso descrito na paciente é bastante comum na rotina clínica veterinária, sendo importante para o diagnóstico de urolitíase e hidrometra a correta anamnese, exame físico e complementar. Em cães é comum encontrar cálculos de oxalato de cálcio e estruvita, sendo a reolução cirúrgica dos urólitos necessária em alguns casos, sendo importante o diagnóstico precoce da cistolitíase para o prognóstico ser favorável. Em cadelas é importante a realização da OSH principalmente em animais jovens para prevenção da hidrometra e posteriormente piometra. O prognóstico é favorável mediante o diagnóstico precocemente.

\section{Referências bibliográficas}

Bartges, J. W. (2012). Chronic kidney disease in dogs and cats. Veterinary Clinics: Small Animal Practice, 42(4):669-692.

Ettinger, S. J., Fedlman, E. C. \& Taibo, R. A. (2002). Tratado de medicina interna veterinaria: enfermedades del perro y el gato. São Paulo, São Paulo, Brasil: Manole.

Ettinger, S. J., Feldman, E. C. \& Cote, E. (2017). Textbook of Veterinary Internal Medicine-eBook. USA: Elsevier Health Sciences.

Fossum, T. W. (2014). Cirurgia de pequenos animais (4 ed. Vol. 1). São Paulo: Elsevier Brasil.

Grauer, G. (2015). Manifestações clínicas dos distúrbios urinários; Urolítiase canina. In A. W. Nelson \& C. G. Couto (Eds.), Medicina Interna de Pequenos Animais. Rio de Janeiro: Elsevier Editora.

Grauer, G. F. (2005). Canine glomerulonephritis: new thoughts on proteinuria and treatment. Journal of Small Animal Practice, 46(10):469-478. 
Houston, D. M., Moore, A. E. P., Favrin, M. G. \& Hoff, B. (2004). Canine urolithiasis: a look at over 16000 urolith submissions to the Canadian Veterinary Urolith Centre from February 1998 to April 2003. The Canadian Veterinary Journal, 45(3):225-230.

Koehler, L. A., Osborne, C. A., Buettner, M. T., Lulich, J. P. \& Behnke, R. (2009). Canine uroliths: frequently asked questions and their answers. Veterinary Clinics of North America: Small Animal Practice, 39(1):161-181.

Latney, L. T. \& Donnelly, T. M. (2013). Urolithiasis. In J. Mayer \& T. Donnelly (Eds.), Clinical veterinary advisor, birds and exotic pets (pp. 282-283). USA: Edit: Saunders.

Ling, G. V., Ruby, A. L., Johnson, D. L., Thurmond, M. \& Franti, C. E. (1998). Renal calculi in dogs and cats: prevalence, mineral type, breed, age, and gender interrelationships (1981-1993). Journal of Veterinary Internal Medicine, 12(1):11-21.

Mialot, J. P. (1984). Patologia da reprodução dos carnívoros domésticos (Vol. 1). Porto Alegre: A Hora veterinária.

Nascimento, E. F. \& Santos, R. L. (2000). Patologia da reprodução dos animais domésticos. Rio de Janeiro: Grupo Gen-Guanabara Koogan.

Nelson, R. W. \& Couto, C. G. (2015). Medicina interna de pequenos animais. Amsterdan: Elsevier Editora.

Oliveira, P. C. d., Lopes, M. D., Thomé, H. E. \& Balieiro, J. C. C. (2008). Avaliação citológica, histológica e hormonal de cadelas normais com complexo hiperplasia endometrial cística/piometra. Veterinária e Zootecnia, 15(1):150-159.

Osborne, C. A., Lulich, J. P., Bartges, J. W., Unger, L. K., Thumchai, R., Koehler, L. A., . . Felice, L. J. (1995). Canine and feline urolithiasis: relationship of etiopathogenesis to treatment and prevention. Canine and feline nephrology and urology, 37(12):798-888.

Osborne, C. A., Lulich, J. P., Polzin, D. J., Sanderson, S. L., Koehler, L. A., Ulrich, L. K., . . Sudo, S. Z. (1999a). Analysis of 77,000 canine uroliths: perspectives from the Minnesota Urolith Center. Veterinary Clinics of North America: Small Animal Practice, 29(1):17-38.

Osborne, C. A., Sanderson, S. L., Lulich, J. P., Bartges, J. W., Ulrich, L. K., Koehler, L. A., . . Swanson, L. L. (1999b). Canine cystine urolithiasis: cause, detection, treatment, and prevention. Veterinary Clinics of North America: Small Animal Practice, 29(1):193-211.

Raditic, D. M. (2015). Complementary and integrative therapies for lower urinary tract diseases. Veterinary Clinics: Small Animal Practice, 45(4):857-878.

Stone, E. A. (2007). Ovário e útero. In R. D. Slatter (Ed.), Manual de cirurgia e pequenos animais. São Paulo: Manole.

Syme, H. M., Markwell, P. J., Pfeiffer, D. \& Elliott, J. (2006). Survival of cats with naturally occurring chronic renal failure is related to severity of proteinuria. Journal of Veterinary Internal Medicine, 20(3):528-535.

Waki, M. F., Ribas Martorelli, C., Erdmann Mosko, P. \& Kogika, M. M. (2010). Classificação em estágios da doença renal crônica em cães e gatos: abordagem clínica, laboratorial e terapêutica. Ciência Rural, 40(10):1-9.

Recebido: 17 de setembro, 2019.

Aprovado: 10 de novembro, 2019.

Publicado: 29 de fevereiro, 2020.

Licenciamento: Este artigo é publicado na modalidade Acesso Aberto sob a licença Creative Commons Atribuição 4.0 (CC-BY 4.0), a qual permite uso irrestrito, distribuição, reprodução em qualquer meio, desde que o autor e a fonte sejam devidamente creditados. 